\title{
Therapeutic benefits of glutamine: An umbrella review of meta-analyses
}

\author{
MARC P. McRAE \\ Department of Basic Science, National University of Health Sciences, Lombard, IL 60148, USA
}

Received February 8, 2017; Accepted March 9, 2017

DOI: 10.3892/br.2017.885

\begin{abstract}
Glutamine may be an essential amino acid in patients with catabolic disease, as it has been demonstrated that circulating glutamine levels drop during critical illness and following major surgery; this may result in an increase in secondary infection risk, recovery time and mortality rates. However, there is much discrepancy in the literature with regards to randomized controlled studies, and therefore, the present study is an umbrella review of published meta-analyses, conducted to examine the effectiveness of glutamine's role as a therapeutic agent. A search using PubMed, Cochrane Library and CINAHL from January 1st, 1980 to December 31st, 2016 was conducted using the following strategy: 'Glutamine AND (meta-analysis OR systematic review)' and publications were retrieved, which provided quantitative statistical analysis of pooled treatment effects on the relative risks of infectious complications, mortality and length of stay in hospital. A total of 22 meta-analyses were entered into the current umbrella review. As displayed in Tables I, II and III, these analyses are split into three groups, based on different parameters. Of the 19 meta-analyses investigating the effects of infectious complications, 15 identified statistically significant reductions in complications, with relative risks ranging between 0.42 and 0.93 . In addition, 12 of the 18 meta-analyses analyzing the length of hospital stays presented statistically significant reductions in the length of stay, with reductions ranging between 0.19 to 4.73 days. Only 4 of the 15 meta-analyses studying mortality effects identified statistically significant reductions in mortality with relative risks ranging between 0.64 and 1.28. Statistically significant heterogeneity was observed in 16 of 22 meta-analyses, and publication bias was observed in five of 11 meta-analyses. Glutamine supplementation for critically ill or surgical patients through parenteral or enteral routes appears to reduce the rate of hospital acquired infectious complications
\end{abstract}

Correspondence to: Dr Marc P. McRae, Department of Basic Science, National University of Health Sciences, 200 E. Roosevelt Road, Lombard, IL 60148, USA

E-mail:mmcrae@nuhs.edu

Key words: glutamine, meta-analysis, critically ill patients, surgery patients, infections and shortening of the length of stay in hospital. Furthermore, glutamine supplementation appeared to reduce the rate of in-patient mortality, but the majority of meta-analyses did not reach statistical significance. However, researchers must appreciate the positive results with caution in light of the fact that there exists statistically significant heterogeneity for the majority of meta-analyses, and statistically significant publication bias in almost half.

\section{Introduction}

Glutamine may be a conditionally essential amino acid in patients with catabolic disease as it has been demonstrated that circulating plasma glutamine concentrations drop during critical illness and following major surgery (1). This drop may be due to glutamine's central role in nitrogen transport within the body, with glutamine contributing to both precursors for nucleic acid synthesis, as well as antioxidant defenses through the production of glutathione (2). In addition, glutamine is the preferred fuel for rapidly dividing cells, such an enterocytes in the small intestine and immune cells, such as lymphocytes, monocytes and macrophages. For some patients, the synthesis and release of glutamine from skeletal muscle is insufficient to meet demands, and a deficiency in glutamine may lead to small intestine mucosal injury followed by increased wall permeability and bacterial translocation (3). These negative effects, along with immunosuppression, may all contribute to an increased probability of secondary infection risk, which may impede the recovery time of the patient, or worse, increase in-patient mortality rates.

More than a dozen clinical studies have suggested that the provision of parenteral or enteral glutamine supplementation in both critically ill and surgical patients may improve nitrogen balance, constitutive protein levels and improve immune function, while decreasing infection rates, the length of stay in hospital and mortality rates (4). However, there is much discrepancy regarding comparing single randomized controlled trials on glutamine supplementation's effects on clinical endpoints such as infection rates, length of stay in hospital and mortality $(5,6)$. This discrepancy may be due to differences in study design, selected patient populations, severity of the disease, nutritional status of the patient and/or the differences in glutamine supplementation relative to different forms (free or dipeptide form), doses and methods of administration (parenteral or enteral). 
Given the inconsistency of the existing literature and the insufficient statistical power due to small sample sizes, a pooling of information from individual trials may provide a more precise and accurate estimate of glutamine's role in reducing the rates of infectious complications and mortality. To achieve this result, many investigators have turned to performing a powerful statistical method known as meta-analysis. Meta-analyses are fundamental to provide the highest level of evidence to best inform health care decision-making. Therefore, the purpose and objective of the present paper was to summarize the evidence from previously published meta-analyses regarding the effectiveness of glutamine's role as a therapeutic dietary agent to reduce the occurrence of infectious complications, length of stay in hospital and mortality.

\section{Materials and methods}

An umbrella review was selected for the current study. An umbrella review provides a summary of existing published meta-analyses and systematic reviews and determines whether authors addressing similar review questions independently observe similar results and arrive at similar conclusions (7).

A systematic literature search of PubMed,Cochrane Library and CINAHL from January 1st, 1980 to December 31st, 2016 was conducted using the following search strategy: 'Glutamine AND (meta-analysis OR systematic review)'. Only English language publications were retrieved, which provided quantitative statistical analysis of pooled treatment effects on the relative risks and odds ratios of infectious complications, length of stay in hospital and mortality of critically ill and surgical patients. Meta-analyses or systematic reviews that did not present study specific summary data using a minimum of four randomized controlled trials or which were focused on infants and children were excluded.

For the published papers that were accepted into the review, the following information was extracted and entered into a spreadsheet: Number of publications included in the meta-analysis, number of total subjects, glutamine dose and method of administration, pooled treatment effects on the relative risks and odds ratios of infectious complications, length of stay in hospital and mortality. In addition, papers were assessed for their disclosure of quality assessment, statistical heterogeneity (Cochrane's Q test and $\mathrm{I}^{2}$ statistic) and publication bias (visual inspection of funnel plots and Egger's or Begg's regression test). As this is a descriptive summary review of meta-analyses, no statistical analyses were performed.

\section{Results}

The initial search strategy identified 106 articles and, following careful review, 26 meta-analyses were retrieved for inclusion into this umbrella review. There were four meta-analyses that were excluded because two were not published in English $(8,9)$, one fell outside the selection criteria, as it was focused on preterm infants (10) and one lacked the necessary methodology and presentation of a well-constructed meta-analysis (11). A flow chart of the meta-analyses selection process is summarized in Fig. 1, and Tables I, II and III provide the detailed analysis of the 22 meta-analyses entered into the umbrella review (12-33).
The results of 19 meta-analyses in Table I indicate that parenteral and/or enteral intakes of 0.3 to $0.45 \mathrm{~g} / \mathrm{kg} / \mathrm{day}$ can provide statistically significantly reductions in the incidence of infectious complications for 15 of the 19 meta-analyses with relative risks of all 19 ranging between 0.42 and 0.93 . Statistically significant heterogeneity was only observed in two of the 19 meta-analyses, however publication bias was observed in four of the nine meta-analyses that chose to use a funnel plot analysis and/or Egger or Begg's regression tests. Fig. 2 displays the bar graph that represents the frequency of the meta-analyses relative rates for the incidence of infectious complications.

The results of 18 meta-analyses in Table II demonstrate that parenteral and/or enteral intakes of 0.3 to $0.45 \mathrm{~g} / \mathrm{kg} / \mathrm{day}$ can provide statistically significantly reductions in the length of stay in hospital for 12 of the 18 meta-analyses. The range for the reduction in length of stay in hospital for all 12 meta-analyses was between 0.19 to 4.73 days. However, statistically significant heterogeneity was only observed in 16 of the 18 meta-analyses. Publication bias was observed in only one of the nine meta-analyses, which chose to use either funnel plot analysis and/or Egger or Begg's regression test. Fig. 3 displays the bar graph that represents the frequency of the meta-analyses average reduction in length of stay in hospital.

The results of 15 meta-analyses in Table III indicate that parenteral and/or enteral intakes of 0.3 to $0.42 \mathrm{~g} / \mathrm{kg} / \mathrm{day}$ provided statistically significant reductions in mortality for only four of the 15 meta-analyses with relative risk for all 15 meta-analyses ranging between 0.64 to 1.28 . Statistically significant heterogeneity was not observed in any of the 15 meta-analyses, and publication bias was observed in only one of the 10 meta-analyses, which chose to use funnel plot analysis and/or Egger or Begg's regression test. Fig. 4 displays the bar graph that represents the frequency of the meta-analyses relative rates for mortality.

\section{Discussion}

With regards to the incidence of infectious complications among critically ill and surgical patients, 15 of the 19 meta-analyses (79\%) presented statistically significant reductions. The rate of infection for all 19 meta-analyses demonstrated a reduction ranging from 7 to $58 \%$. Just over half of the meta-analyses (10 of 19) identified reductions of between 10 and 30\%, and one-third of the meta-analyses (seven of 19) found reduction of between 30 and $50 \%$. It should be noted that four of the nine meta-analyses presented with significant publication bias, but more troubling is the fact that only nine of the 19 meta-analyses (47\%) assessed for publication bias. Publication bias occurs because small studies with null results tend not to be published, and this problem is referred to as the 'file drawer problem'. As published studies are more likely to report positive research outcomes than unpublished ones, the significance of the effect size of the weighted average of the published studies is overestimated, and this can potentially bias the results of the meta-analysis. On a positive note, only one of the 15 meta-analyses that identified statistically significant reductions in infectious complications presented with statistically significant heterogeneity. 
Table I. Summary of the meta-analyses on infectious complications that qualified for the present umbrella review on glutamine.

\begin{tabular}{|c|c|c|c|c|c|c|c|c|c|}
\hline $\begin{array}{l}\text { Meta-analysis } \\
\text { authors } \\
\text { and date }\end{array}$ & $\begin{array}{l}\text { Patient } \\
\text { type }\end{array}$ & $\begin{array}{l}\text { Number } \\
\text { of } \\
\text { studies } \\
\text { in meta- } \\
\text { analysis }\end{array}$ & $\begin{array}{l}\text { Number } \\
\text { of } \\
\text { subjects } \\
\text { in meta } \\
\text { analysis }\end{array}$ & $\begin{array}{l}\text { Average } \\
\text { glutamine } \\
\text { dose and } \\
\text { admin- } \\
\text { istration }\end{array}$ & $\begin{array}{l}\text { Main } \\
\text { findings } \\
\text { of meta- } \\
\text { analysis } \\
\text { RR and } \\
\text { P-value }\end{array}$ & $\begin{array}{c}\text { Q-test } \\
\text { P-value }\end{array}$ & $\begin{array}{c}\mathrm{I}^{2} \\
\text { statistic } \\
\%\end{array}$ & $\begin{array}{l}\text { Funnel } \\
\text { plot or } \\
\text { Egger or } \\
\text { Begg's } \\
\text { test } \\
\text { P-value }\end{array}$ & (Refs.) \\
\hline Novak et al 2002 & Mixed & 7 & 326 & $\begin{array}{c}0.30 \\
\mathrm{PN} \text { and } \mathrm{EN}\end{array}$ & $\begin{array}{c}0.80 \\
\mathrm{P}=0.03\end{array}$ & 0.43 & NR & & (12) \\
\hline Avenell 2006 & Mixed & 19 & 1,370 & NR & $\begin{array}{c}0.76 \\
\mathrm{P}=0.002\end{array}$ & 0.26 & 16 & 0.03 & (13) \\
\hline Avenell 2009 & Mixed & 24 & 1,776 & $\begin{array}{c}0.42 \\
\text { PN and EN }\end{array}$ & $\begin{array}{c}0.81 \\
\mathrm{P}=0.003\end{array}$ & 0.06 & 33 & $\begin{array}{c}\text { +ve } \\
\text { funnel } \\
\text { plot }\end{array}$ & $(14)$ \\
\hline Bollhalder et al 2013 & Mixed & 30 & 2,014 & $\begin{array}{c}0.42 \\
\text { PN and EN }\end{array}$ & $\begin{array}{c}0.83 \\
\mathrm{P}=0.009\end{array}$ & 0.22 & 17 & 0.36 & $(15)$ \\
\hline Chen et al 2014 & Mixed & 15 & 2,862 & $\begin{array}{c}0.40 \\
\mathrm{PN} \text { and } \mathrm{EN}\end{array}$ & $\begin{array}{c}0.85 \\
\mathrm{P}=0.02\end{array}$ & 0.01 & 51 & NS & $(16)$ \\
\hline Crowther et al 2009 & Critically ill & 7 & 274 & $\begin{array}{c}0.40 \\
\mathrm{PN} \text { and } \mathrm{EN}\end{array}$ & $\begin{array}{c}0.77 \\
\mathrm{P}=0.03\end{array}$ & 0.46 & 0 & & $(17)$ \\
\hline Asrani et al 2013 & Critically ill & 8 & 365 & $\begin{array}{c}0.36 \\
\mathrm{PN} \text { and } \mathrm{EN}\end{array}$ & $\begin{array}{c}0.58 \\
\mathrm{P}=0.009\end{array}$ & 0.29 & 18 & & (18) \\
\hline Zhong et al 2013 & Critically ill & 4 & 186 & $\begin{array}{c}0.40 \\
\text { PN and EN }\end{array}$ & $\begin{array}{c}0.59 \\
\mathrm{P}=0.006\end{array}$ & 0.37 & 4 & & $(19)$ \\
\hline Tao et al 2014 & Critically ill & 33 & 2,303 & $\begin{array}{c}0.38 \\
\mathrm{PN} \text { and } \mathrm{EN}\end{array}$ & $\begin{array}{c}0.79 \\
\mathrm{P}<0.000\end{array}$ & NR & 8 & $\begin{array}{c}\text { +ve } \\
\text { funnel } \\
\text { plot }\end{array}$ & $(20)$ \\
\hline Wischmeyer et al 2014 & Critically ill & 12 & 1,264 & $\begin{array}{c}0.35 \\
\text { PN and EN }\end{array}$ & $\begin{array}{c}0.86 \\
\mathrm{P}=0.09\end{array}$ & 0.06 & 43 & 0.05 & $(21)$ \\
\hline Oldani et al 2015 & Critically ill & 15 & 2,795 & $\begin{array}{c}0.38 \\
\text { PN and EN }\end{array}$ & $\begin{array}{c}0.88 \\
\mathrm{P}=0.11\end{array}$ & 0.004 & 56 & NS & $(22)$ \\
\hline Yong et al 2016 & Critically ill & 6 & 243 & $\begin{array}{c}\text { NR } \\
\text { PN and EN }\end{array}$ & $\begin{array}{c}0.62 \\
\mathrm{P}=0.002\end{array}$ & 0.45 & 0 & 0.093 & (23) \\
\hline van Zanten et al 2015 & Critically ill & 5 & 776 & $\begin{array}{c}0.38 \\
\text { EN only }\end{array}$ & $\begin{array}{c}0.93 \\
\mathrm{P}=0.39\end{array}$ & 0.40 & 0 & 0.23 & $(24)$ \\
\hline Jiang et al 2004 & Surgical & 10 & 355 & $\begin{array}{c}0.45 \\
\text { PN only }\end{array}$ & $\begin{array}{c}0.42 \\
\mathrm{P}=0.002\end{array}$ & 0.97 & NR & & $(25)$ \\
\hline Zheng et al 2006 & Surgical & 5 & 215 & $\begin{array}{c}0.32 \\
\text { PN only }\end{array}$ & $\begin{array}{c}0.76 \\
\mathrm{P}=0.04\end{array}$ & 0.94 & NR & & $(26)$ \\
\hline Wang et al 2010 & Surgical & 10 & 447 & $\begin{array}{c}0.34 \\
\text { PN only }\end{array}$ & $\begin{array}{c}0.59 \\
\mathrm{P}=0.003\end{array}$ & 0.65 & 0 & & (27) \\
\hline Yue et al 2013 & Surgical & 13 & 653 & $\begin{array}{c}0.31 \\
\text { PN only }\end{array}$ & $\begin{array}{c}0.52 \\
\mathrm{P}=0.002\end{array}$ & 0.83 & 0 & & $(28)$ \\
\hline Kang et al 2015 & Surgical & 8 & 872 & $\begin{array}{c}0.34 \\
\text { PN only }\end{array}$ & $\begin{array}{c}0.67 \\
\mathrm{P}=0.008\end{array}$ & 0.06 & 49 & & (29) \\
\hline Sandini et al 2015 & Surgical & 13 & 1,011 & $\begin{array}{c}0.37 \\
\text { PN and EN }\end{array}$ & $\begin{array}{c}0.64 \\
\mathrm{P}=0.087\end{array}$ & 0.14 & 32 & & $(30)$ \\
\hline
\end{tabular}

EN, enteral; NR, not reported; NS, not significant; PN, parenteral; RR, relative risk. 
Table II. Summary of the meta-analyses on length of stay in hospital that qualified for the umbrella review on glutamine.

\begin{tabular}{|c|c|c|c|c|c|c|c|c|c|}
\hline $\begin{array}{l}\text { Meta-analysis } \\
\text { authors } \\
\text { and date }\end{array}$ & $\begin{array}{l}\text { Patient } \\
\text { type }\end{array}$ & $\begin{array}{l}\text { Number } \\
\text { of } \\
\text { studies } \\
\text { in meta- } \\
\text { analysis }\end{array}$ & $\begin{array}{l}\text { Number } \\
\text { of } \\
\text { subjects } \\
\text { in meta } \\
\text { analysis }\end{array}$ & $\begin{array}{l}\text { Average } \\
\text { glutamine } \\
\text { dose and } \\
\text { admin- } \\
\text { istration }\end{array}$ & $\begin{array}{l}\text { Reduction } \\
\text { of } \\
\text { hospital } \\
\text { length } \\
\text { of stay } \\
\text { in days }\end{array}$ & $\begin{array}{l}\text { Q-test } \\
\text { P-value }\end{array}$ & $\begin{array}{c}\mathrm{I}^{2} \\
\text { statistic } \\
\%\end{array}$ & $\begin{array}{l}\text { Funnel } \\
\text { plot or } \\
\text { Egger or } \\
\text { Begg's } \\
\text { test } \\
\text { P-value }\end{array}$ & (Refs.) \\
\hline Novak et al 2002 & Mixed & 10 & 541 & $\begin{array}{c}0.30 \\
\text { PN and EN }\end{array}$ & $\begin{array}{c}2.6 \\
\mathrm{P}<0.05\end{array}$ & 0.002 & NR & & (12) \\
\hline $\begin{array}{l}\text { Bollhalder } \text { et al } \\
2013\end{array}$ & Mixed & 30 & 2,109 & $\begin{array}{c}0.42 \\
\text { PN and EN }\end{array}$ & $\begin{array}{c}2.35 \\
\mathrm{P}=0.001\end{array}$ & 0.001 & 86 & 0.54 & (15) \\
\hline Chen et al 2014 & Mixed & 14 & 2,777 & $\begin{array}{c}0.40 \\
\text { PN and EN }\end{array}$ & $\begin{array}{c}1.48 \\
\mathrm{P}=0.24\end{array}$ & $<0.0001$ & 96 & NS & (16) \\
\hline Crowther et al 2009 & $\begin{array}{l}\text { Critically } \\
\text { ill }\end{array}$ & 12 & 552 & $\begin{array}{c}0.40 \\
\text { PN and EN }\end{array}$ & $\begin{array}{l}0.42 \\
\text { NS }\end{array}$ & NR & 36 & & (17) \\
\hline Asrani et al 2013 & $\begin{array}{l}\text { Critically } \\
\quad \text { ill }\end{array}$ & 9 & 390 & $\begin{array}{c}0.36 \\
\text { PN and EN }\end{array}$ & $\begin{array}{c}1.35 \\
\mathrm{P}=0.17\end{array}$ & 0.0001 & 78 & & (18) \\
\hline Lin et al 2013 & $\begin{array}{l}\text { Critically } \\
\text { ill }\end{array}$ & 4 & 155 & $\begin{array}{c}0.44 \\
\text { EN only }\end{array}$ & $\begin{array}{l}3.37 \\
\text { NS }\end{array}$ & 0.02 & NR & & (31) \\
\hline Tao et al 2014 & $\begin{array}{l}\text { Critically } \\
\text { ill }\end{array}$ & 36 & 2,963 & $\begin{array}{c}0.38 \\
\text { PN and EN }\end{array}$ & $\begin{array}{c}3.46 \\
\mathrm{P}<0.0001\end{array}$ & NR & 63 & NS & (20) \\
\hline $\begin{array}{l}\text { Wischmeyer } \text { et al } \\
2014\end{array}$ & $\begin{array}{l}\text { Critically } \\
\text { ill }\end{array}$ & 11 & 639 & $\begin{array}{c}0.35 \\
\text { PN and EN }\end{array}$ & $\begin{array}{c}2.56 \\
\mathrm{P}=0.02\end{array}$ & 0.002 & 63 & 0.87 & (21) \\
\hline Oldani et al 2015 & $\begin{array}{l}\text { Critically } \\
\text { ill }\end{array}$ & 19 & 2,635 & $\begin{array}{c}0.38 \\
\text { PN and EN }\end{array}$ & $\begin{array}{c}1.73 \\
\mathrm{P}=0.09\end{array}$ & 0.02 & 44 & NS & (22) \\
\hline Yong et al 2016 & $\begin{array}{l}\text { Critically } \\
\quad \text { ill }\end{array}$ & 7 & 303 & $\begin{array}{c}\text { NR } \\
\text { PN and EN }\end{array}$ & $\begin{array}{c}3.89 \\
\mathrm{P}<0.0001\end{array}$ & 0.38 & 6 & 0.76 & (23) \\
\hline $\begin{array}{l}\text { van Zanten et al } \\
2015\end{array}$ & $\begin{array}{l}\text { Critically } \\
\text { ill }\end{array}$ & 7 & 583 & $\begin{array}{c}0.38 \\
\text { EN only }\end{array}$ & $\begin{array}{c}4.73 \\
\mathrm{P}=0.02\end{array}$ & 0.05 & 52 & 0.48 & (24) \\
\hline Mottaghi et al 2016 & Criticallyl & 6 & 1,286 & $\begin{array}{c}0.42 \\
\text { EN only }\end{array}$ & $\begin{array}{c}0.19 \\
\text { NS }\end{array}$ & $<0.01$ & 95 & $\begin{array}{c}+\mathrm{ve} \\
\text { funnel } \\
\text { plot }\end{array}$ & (32) \\
\hline Jiang et al 2004 & Surgical & 8 & 275 & $\begin{array}{c}0.45 \\
\text { PN only }\end{array}$ & $\begin{array}{c}3.25 \\
\mathrm{P}=0.0001\end{array}$ & 0.0003 & NR & & (25) \\
\hline Zheng et al 2006 & Surgical & 6 & 291 & $\begin{array}{c}0.32 \\
\text { PN only }\end{array}$ & $\begin{array}{c}3.55 \\
\mathrm{P}<0.0001\end{array}$ & $<0.0001$ & NR & & (26) \\
\hline Wang et al 2010 & Surgical & 11 & 377 & $\begin{array}{c}0.34 \\
\text { PN only }\end{array}$ & $\begin{array}{c}3.84 \\
\mathrm{P}<0.0001\end{array}$ & $<0.0001$ & 84 & & (27) \\
\hline Yue et al 2013 & Surgical & 12 & 525 & $\begin{array}{c}0.31 \\
\text { PN only }\end{array}$ & $\begin{array}{c}3.33 \\
\mathrm{P}<0.0001\end{array}$ & $<0.0001$ & 88 & & (28) \\
\hline Kang et al 2015 & Surgical & 4 & 729 & $\begin{array}{c}0.34 \\
\text { PN only }\end{array}$ & $\begin{array}{c}1.72 \\
\mathrm{P}=0.03\end{array}$ & $<0.0001$ & 91 & & (29) \\
\hline Sandini et al 2015 & Surgical & 13 & 1,000 & $\begin{array}{c}0.37 \\
\text { PN and EN }\end{array}$ & $\begin{array}{c}2.67 \\
\mathrm{P}<0.0001\end{array}$ & 0.0001 & 86 & NS & (30) \\
\hline
\end{tabular}

EN, enteral; NR, not reported; NS, not significant; PN, parenteral.

In regard to the length of stay in hospital, 12 of the 18 meta-analyses $(67 \%)$ presented statistically significant decreases in which the time in hospital decreased between
0.19 to 4.73 days. There were $\sim$ two-thirds of the meta-analyses (11 of 18) that demonstrated a 2 to 4 day reduction in the length of stay in hospital. However, it should be noted that 16 of the 
Table III. Summary of the meta-analyses on mortality that qualified for this umbrella review on glutamine.

\begin{tabular}{|c|c|c|c|c|c|c|c|c|c|}
\hline $\begin{array}{l}\text { Meta-analysis } \\
\text { authors } \\
\text { and date }\end{array}$ & $\begin{array}{l}\text { Patient } \\
\text { type }\end{array}$ & $\begin{array}{l}\text { Number } \\
\text { of } \\
\text { studies } \\
\text { in meta- } \\
\text { analysis }\end{array}$ & $\begin{array}{l}\text { Number } \\
\text { of } \\
\text { subjects } \\
\text { in meta } \\
\text { analysis }\end{array}$ & $\begin{array}{c}\text { Average } \\
\text { glutamine } \\
\text { dose and } \\
\text { admin- } \\
\text { istration }\end{array}$ & $\begin{array}{l}\text { Main } \\
\text { findings } \\
\text { of meta- } \\
\text { analysis } \\
\text { RR and } \\
\text { P-value }\end{array}$ & $\begin{array}{l}\text { Q-test } \\
\text { P-value }\end{array}$ & $\begin{array}{c}\mathrm{I}^{2} \\
\text { statistic } \\
\%\end{array}$ & $\begin{array}{l}\text { Funnel } \\
\text { plot or } \\
\text { Egger or } \\
\text { Begg's } \\
\text { test } \\
\text { P-value }\end{array}$ & (Refs.) \\
\hline Novak et al 2002 & Mixed & 14 & 751 & $\begin{array}{c}0.30 \\
\text { PN and EN }\end{array}$ & $\begin{array}{l}0.78 \\
\text { NS }\end{array}$ & 0.99 & NR & & (12) \\
\hline Avenell 2006 & Mixed & 15 & 1,449 & NR & $\begin{array}{c}0.81 \\
\mathrm{P}=0.07\end{array}$ & 0.33 & 11 & NS & (13) \\
\hline Avenell 2009 & Mixed & 21 & 1,964 & $\begin{array}{c}0.42 \\
\text { PN and EN }\end{array}$ & $\begin{array}{c}0.84 \\
\mathrm{P}=0.17\end{array}$ & 0.16 & 23 & NS & (14) \\
\hline $\begin{array}{l}\text { Bollhalder } \text { et al } \\
2013\end{array}$ & Mixed & 22 & 2,126 & $\begin{array}{c}0.42 \\
\text { PN and EN }\end{array}$ & $\begin{array}{c}0.89 \\
\mathrm{P}=0.15\end{array}$ & 0.53 & 0 & 0.61 & (15) \\
\hline Chen et al 2014 & Mixed & 17 & 3,383 & $\begin{array}{c}0.40 \\
\text { PN and EN }\end{array}$ & $\begin{array}{c}1.01 \\
\mathrm{P}=0.87\end{array}$ & 0.26 & 17 & NS & (16) \\
\hline Heyland et al 2003 & $\begin{array}{l}\text { Critically } \\
\text { ill }\end{array}$ & 4 & 397 & NR & $\begin{array}{c}0.71 \\
\mathrm{P}=0.04\end{array}$ & 0.91 & NR & & (33) \\
\hline $\begin{array}{l}\text { Crowther et al } \\
2009\end{array}$ & $\begin{array}{c}\text { Critically } \\
\text { ill }\end{array}$ & 8 & 572 & $\begin{array}{c}0.40 \\
\text { PN and EN }\end{array}$ & $\begin{array}{c}0.88 \\
\mathrm{P}=0.49\end{array}$ & 0.45 & 0 & & (17) \\
\hline Asrani et al 2013 & $\begin{array}{c}\text { Critically } \\
\text { ill }\end{array}$ & 9 & 380 & $\begin{array}{c}0.36 \\
\text { PN and EN }\end{array}$ & $\begin{array}{c}0.70 \\
\mathrm{P}=0.001\end{array}$ & 0.99 & 0 & & (18) \\
\hline Zhong et al 2013 & $\begin{array}{c}\text { Critically } \\
\quad \text { ill }\end{array}$ & 4 & 190 & $\begin{array}{c}0.40 \\
\text { PN and EN }\end{array}$ & $\begin{array}{c}0.74 \\
\mathrm{P}=0.01\end{array}$ & 0.84 & 0 & & (19) \\
\hline Tao et al 2014 & $\begin{array}{c}\text { Critically } \\
\text { ill }\end{array}$ & 36 & 3,454 & $\begin{array}{c}0.38 \\
\text { PN and EN }\end{array}$ & $\begin{array}{c}0.89 \\
\mathrm{P}=0.10\end{array}$ & NR & 22 & $\begin{array}{c}+\mathrm{ve} \\
\text { funnel } \\
\text { plot }\end{array}$ & (20) \\
\hline $\begin{array}{l}\text { Wischmeyer et al } \\
2014\end{array}$ & $\begin{array}{c}\text { Critically } \\
\quad \text { ill }\end{array}$ & 24 & 2,312 & $\begin{array}{c}0.35 \\
\text { PN and EN }\end{array}$ & $\begin{array}{c}0.88 \\
\mathrm{P}=0.10\end{array}$ & 0.58 & 0 & 0.57 & (21) \\
\hline Oldani et al 2015 & $\begin{array}{c}\text { Critically } \\
\text { ill }\end{array}$ & 24 & 2,834 & $\begin{array}{c}0.38 \\
\text { PN and EN }\end{array}$ & $\begin{array}{c}0.93 \\
\mathrm{P}=0.33\end{array}$ & 0.31 & 11 & NS & (22) \\
\hline Yong et al 2016 & $\begin{array}{c}\text { Critically } \\
\text { ill }\end{array}$ & 6 & 243 & $\begin{array}{c}\text { NR } \\
\text { PN and EN }\end{array}$ & $\begin{array}{c}0.64 \\
\mathrm{P}=0.02\end{array}$ & 0.93 & 0 & 0.09 & (23) \\
\hline $\begin{array}{l}\text { van Zanten et al } \\
2015\end{array}$ & $\begin{array}{c}\text { Critically } \\
\text { ill }\end{array}$ & 10 & 1,022 & $\begin{array}{c}0.38 \\
\text { EN only }\end{array}$ & $\begin{array}{c}0.94 \\
\mathrm{P}=0.74\end{array}$ & 0.26 & 21 & 0.18 & (24) \\
\hline $\begin{array}{l}\text { Mottaghi et al } \\
2016\end{array}$ & $\begin{array}{c}\text { Critically } \\
\text { ill }\end{array}$ & 8 & 1,327 & $\begin{array}{c}0.42 \\
\text { EN only }\end{array}$ & $\begin{array}{c}1.28 \\
\mathrm{P}=0.07\end{array}$ & 0.62 & 0 & NS & (32) \\
\hline
\end{tabular}

EN, enteral; NR, not reported; NS, not significant; PN, parenteral; RR, relative risk.

18 meta-analyses $(89 \%)$ presented with statistically significant heterogeneity and this weakens the clinical certainty of this effect. Ideally the studies combined into any given meta-analysis should all have used the same experimental protocols; however increased heterogeneity is inevitable due to the wide variation in study design. Differences in study design include number of subjects, age, sex, body mass index, nutritional status (plasma glutamine concentration at admission), total energy intake, type of disease or surgery, disease severity, dosage, timing, duration, chemical form
(L-glutamine, alanyl-L-glutamine or glycyl-L-glutamine) and route of glutamine administration (parenteral vs. enteral). On a positive note, only one of the nine meta-analyses presented with statistically significant publication bias, but again this is problematic as only nine of the 18 meta-analyses (50\%) assessed for publication bias.

In regard to mortality rate, 10 of the 15 meta-analyses $(67 \%)$ identified a reduction that ranged between 10 and $30 \%$, however, only four of the 15 meta-analyses (27\%) demonstrated statistically significant reductions. More troubling is 


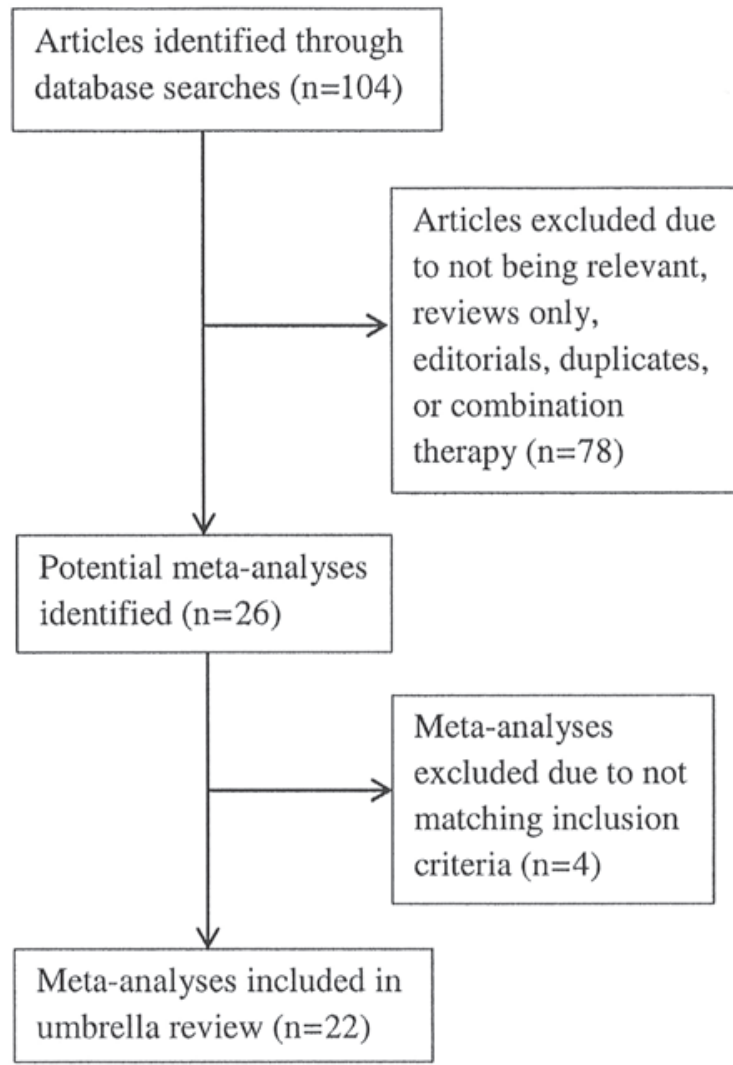

Figure 1. Flow chart of meta-analysis selection.

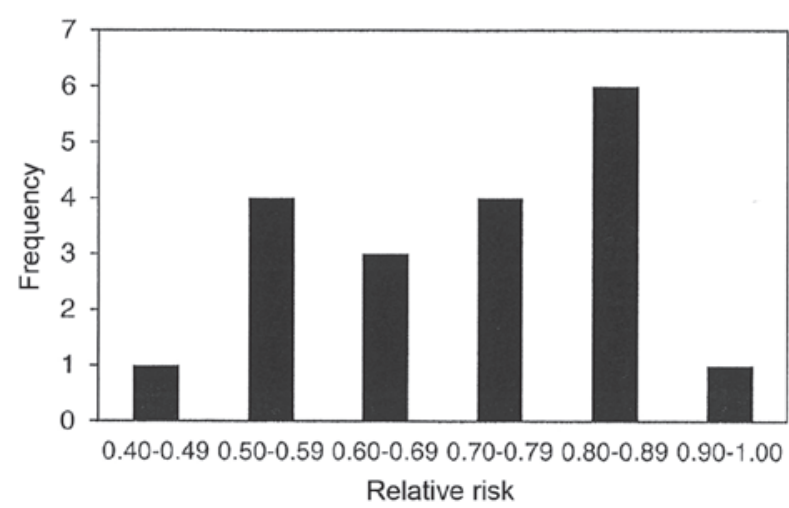

Figure 2. Frequency of the meta-analyses relative rates for the incidence of infectious complications.

the fact that these four meta-analyses represented four of the six that possessed $<10$ clinical studies in their make-up $(4,4,6$ and 9 respectively). Meanwhile, nine of the 11 meta-analyses with non-significant findings were made up of between 10 to 36 clinical studies, and so there appears to be a bias towards meta-analyses presenting statistical significance when using $<10$ clinical trials. This relationship was not observed in the infectious complications or length of stay in hospital umbrella reviews. However, heterogeneity and publication bias was never found to be an issue, as none of the meta-analyses presented statistically significant heterogeneity, and only one of the 10 meta-analyses suggested a potential for publication bias.

The apparent effect of glutamine supplementation to reduce the incidence of infectious complications in critically ill and

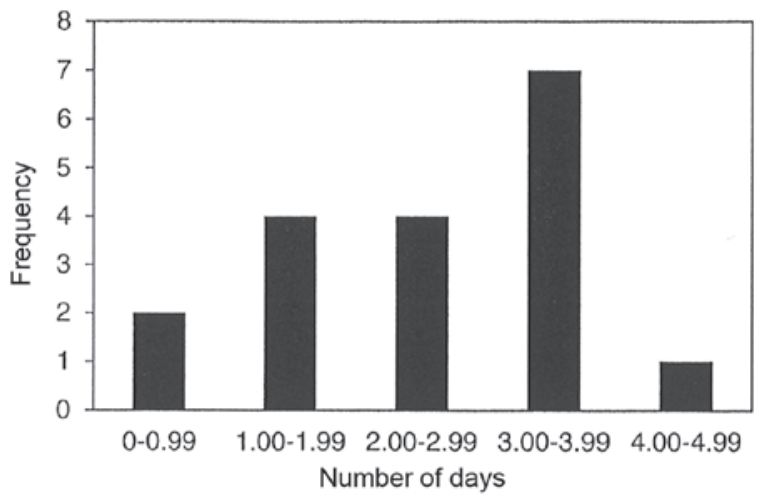

Figure 3. Frequency of the meta-analyses average reduction in length of stay in hospital.

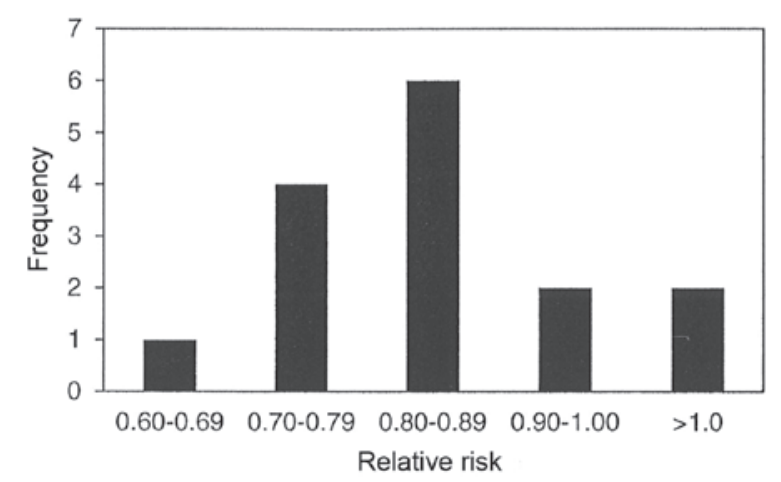

Figure 4. Frequency of the meta-analyses relative rates for mortality.

surgical patients is probably due to the fact that glutamine is the preferred fuel for enterocytes for maintaining gastrointestinal structure and decreasing gut mucosal atrophy, which could otherwise lead to increased intestinal permeability and bacterial translocation (3). In addition, glutamine positively affects lymphocyte proliferation, cytokine production, macrophage phagocytosis and the secretion of intestinal immune globulin $\operatorname{Ig} \mathrm{A}$, which further reduces bacterial adhesion and prevents translocation (34). Therefore, glutamine serves a critical role in the protection of the intestinal immune barrier and leads to increased resistance to bacterial translocation and, hence, reduces infectious complication rates. This fact is supported by a meta-analysis that presented beneficial changes in the markers of intestinal inflammation and mucosal permeability with abdominal surgery patients (35). Finally, glutamine supplementation enhances cellular heat shock protein production (HSP70 and HSP72) and glutathione expression, which both protect cells and enhance cell survival. Heat shock proteins are involved in the repair and removal of damaged proteins, and glutathione reacts directly with reactive oxygen species in order to prevent oxidative damage (36). Collectively, both mechanisms of action reduce the likelihood of cellular apoptosis.

If glutamine supplementation can reduce the incidence of hospital acquired infectious complications, then, when averaged over a large number of patients, this can result in a shorted length of stay in hospital. If a critically ill or surgical patient does acquire an infectious complication, this will prolong that patient's length of stay in hospital and require 
more resources. Hospital acquired infections are expensive to treat, and it is estimated that hospital acquired infections can prolong a patient's length of stay by at least 4 days; this can have a significant financial impact on hospitalization cost (27). It has been observed that the additional cost of supplemented glutamine treatment is more than completely offset by cost savings in hospital care (37).

Low plasma glutamine concentrations in patients admitted to hospitals are an independent predictor of premature death, but it is unclear whether the decline of circulating glutamine contributes to death, or whether it is a simple marker of disease severity (38). Assuming that supplemental glutamine's primary mechanism of action is in the prevention of hospital acquired infections, then it is understandable why there were no significant changes observed in mortality rates. To begin with, the mortality rate for patients admitted to hospital as inpatients is $2 \%$ (39). Furthermore, hospital acquired infections occur in $\sim 4 \%$ of all admitted patients and only $\sim 6 \%$ of those patients with hospital acquired infections die during their hospitalization $(40,41)$. From these data, it can be estimated that the mortality rate due to hospital acquired infections for all patients admitted to hospitals is $\sim 0.25 \%$, which represents only an eighth of all in-patient mortality rates. Therefore, for the majority of meta-analyses that did not identify a significant difference in mortality rates, this is probably due to the fact that the majority of clinical studies used in these meta-analyses were too small and too underpowered to detect these small positive changes in mortality rates.

Although enteral glutamine supplementation should benefit intestinal enterocyte function and overall gut barrier structure better than parenteral glutamine supplementation, it has been suggested that there is a greater treatment effect observed for parenteral compared with enteral glutamine supplementation $(12,14,16,18)$. This is thought to be due to the higher bioavailability of glutamine through parenteral administration, whereas enteral glutamine is used by the gut, therefore the immune system outside the gut would not have been affected by enteral glutamine supplementation. However, only one of the eight meta-analyses that performed a subgroup analysis on administration type presented a significant difference between parenteral and enteral administration, and this meta-analysis found that the length of stay in hospital was significantly shortened for the enteral route ( 4.5 days less for enteral vs. 2.5 days less for parenteral) (23). To confuse matters more, the only two meta-analyses on clinical studies that used enteral glutamine supplementation with critically ill patients demonstrated completely opposite effects on length of stay in hospital with one presenting a 4.73 day reduction and the other with a 0.19 day reduction (it should be noted that these two findings represent the two extremes from the 18 meta-analyses in this category) $(24,32)$. Although both of these meta-analyses examined clinical studies using enteral feeding in critically ill patients only, there was only one common clinical study used by both meta-analyses of the combined total of 12 different clinical studies. The fact that there appears no real difference in outcomes between enteral and parenteral should make sense, considering that many of the potential protective mechanisms of glutamine supplementation given either enterally or parenterally overlap and are quite similar.
Four of six meta-analyses that performed subgroup analysis on high vs. low dose glutamine supplementation observed that the mortality rate was lower with high dose compared to low dose glutamine supplementation, but the differences between groups were never statistically significant $(12,14-16,22,30)$. Of the three meta-analyses that performed subgroup analyses comparing critically ill vs. surgical patients, it also appears that there was no significant difference between these two groups in their response to glutamine supplementation $(12,15,16)$.

In regard to side effects, only 11 of the 22 meta-analyses $(50 \%)$ addressed this topic with six of them noting that no side effects were observed $(12-14,19,24,26)$, and the remaining five meta-analyses all mentioned the REDOX study that indicated that critically ill patients with glutamine doses of $>0.5 \mathrm{~g} / \mathrm{kg} /$ day had higher urea concentrations and higher mortality rates $(20-22,32)$. However, it has been noted that there were problems with the REDOX study as $30 \%$ of the patients in the glutamine supplementation group were shown to have baseline renal failure at admission, which is a very common exclusion criteria for the majority of glutamine supplementation clinical trials $(16,21)$. In the REDOX post-hoc analysis the authors of the study concluded that high-dose glutamine supplementation was only associated with increased mortality in critically ill patients with multi organ failure (42).

Using mostly Jadad or Cochrane quality assessment scales, 18 of the 22 meta-analyses obtained quality assessment scores and the average percentages of high and low quality clinical studies used in any given meta-analysis was 30.4 and $13.8 \%$, respectively. Although none of the meta-analyses specified that clinical studies were excluded from their analysis, more recently published meta-analyses may have excluded low quality studies from the outset without specifying that they were excluded. For the majority of the meta-analyses (11 of the 22), the number of clinical studies entered for analysis ranged between 10 and 20 . There were five meta-analyses that used $<6$ clinical studies, and one meta-analysis published in 2014 used 53 clinical studies, and these six analyses represent $43 \%$ of the 125 unique clinical studies used by all 22 meta-analyses (20). Of the 125 unique clinical studies used by the 22 meta-analyses in this umbrella review, 57 (46\%) were used only once. This potentially suggests that there are many clinical studies of such poor quality that they may have made the inclusion criteria by one meta-analysis but excluded by all the others. Or it may simply be due to the fact that a particular meta-analysis may have had a very specific focus [acute pancreatitis $(18,19)$, burn patients $(31)$ or gastrointestinal tumor patients (29)] and therefore the clinical studies used by this meta-analysis would have been specifically selected by this one meta-analysis and no other.

This umbrella review has certain limitations. One limitation is that only three indexing systems were searched, and thus it is possible that some meta-analyses were not identified. Another limitation is that only one author performed the search and selection of the meta-analyses included in this umbrella review. And finally, as in all literature reviews, the quality of this umbrella review is directly related to the quality of the included meta-analyses, which are dependent upon the quality of the individual clinical studies used to conduct the meta-analysis. 
In conclusion, this umbrella review supports the statement by the American Society for Parenteral and Enteral Nutrition that suggests that parenteral glutamine supplementation should probably be given early and in doses between 0.2 and $0.5 \mathrm{~g} / \mathrm{kg} / \mathrm{day}$ (43). This statement is supported by the fact that a majority of the meta-analyses reviewed identified significant positive benefits for glutamine supplementation to reduce the rate of hospital acquired infectious complications, and shortening of the length of stay in hospital. In addition, glutamine supplementation appeared to reduce the rate of hospital mortality, but for the majority of these meta-analyses they did not reach statistical significance. However, it must be appreciated that all these positive results with caution in light of the fact that there exists statistically significant heterogeneity in the length of stay in hospital meta-analyses, and statistically significant publication bias in the infectious complications meta-analyses. Also, the lack of quality assessment of published clinical studies is problematic, as clinical studies of very low quality may have been included in these meta-analyses, which can therefore potentially bias their overall outcomes. Despite the problems of heterogeneity, publication bias and quality assessment, the potential benefits observed in this umbrella review strongly suggests that parenteral and enteral glutamine supplementation as a component of nutritional support can be considered as an approach to improve the outcomes of critically ill and surgical patients. Finally, in regards to determining if there is beneficial effect on reducing hospital mortality rates with glutamine supplementation, more well-designed multicenter randomized controlled trials using large populations are required.

\section{References}

1. Wischmeyer PE: Glutamine: Mode of action in critical illness. Crit Care Med 35 (Suppl 9): S541-S544, 2007.

2. Wischmeyer PE: Glutamine: Role in critical illness and ongoing clinical trials. Curr Opin Gastroenterol 24: 190-197, 2008.

3. Wischmeyer PE: Glutamine: Role in gut protection in critical illness. Curr Opin Clin Nutr Metab Care 9: 607-612, 2006.

4. van Zanten AR: Glutamine and antioxidants: Status of their use in critical illness. Curr Opin Clin Nutr Metab Care 18: 179-186, 2015.

5. Cynober L and De Bandt JP: Glutamine in the intensive care unit. Curr Opin Clin Nutr Metab Care 17: 98-104, 2014.

6. Mundi MS, Shah M and Hurt RT: When is it appropriate to use glutamine in critical illness? Nutr Clin Pract 31: 445-450, 2016.

7. Aromataris E, Fernandez R, Godfrey CM, Holly C, Khalil H and Tungpunkom P: Summarizing systematic reviews: Methodological development, conduct and reporting of an umbrella review approach. Int J Evid Based Healthc 13: 132-140, 2015.

8. Jiang ZM and Jiang H: The clinical efficacy of glutamine dipeptides on postoperative patients: An updated systematic review of randomized controlled trials from Europe and Asia (1997-2005). Zhonghua Yi Xue Za Zhi 86: 1610-1614, 2006 (In Chinese).

9. Jiang H, Chen W, Hu W, Cai B and Liao RJ: The impact of glutamine-enhanced enteral nutrition on clinical outcome of patients with critical illness: A systematic review of randomized controlled trials. Zhonghua Shao Shang Za Zhi 25: 325-330, 2009 (In Chinese)

10. Moe-Byrne T, Brown JV and McGuire W: Glutamine supplementation to prevent morbidity and mortality in preterm infants. Cochrane Database Syst Rev 4: CD001457, 2016.

11. Castro-Gutiérrez V and Rada G: Is there a role for glutamine supplementation in the management of acute pancreatitis? Medwave 16 (Suppl 3): e6512, 2016.

12. Novak F, Heyland DK, Avenell A, Drover JW and Su X: Glutamine supplementation in serious illness: A systematic review of the evidence. Crit Care Med 30: 2022-2029, 2002.

13. Avenell A: Glutamine in critical care: Current evidence from systematic reviews. Proc Nutr Soc 65: 236-241, 2006.
14. Avenell A: Hot topics in parenteral nutrition. Current evidence and ongoing trials on the use of glutamine in critically-ill patients and patients undergoing surgery. Proc Nutr Soc 68: 261-268, 2009.

15. Bollhalder L, Pfeil AM, Tomonaga Y and Schwenkglenks M: A systematic literature review and meta-analysis of randomized clinical trials of parenteral glutamine supplementation. Clin Nutr 32: 213-223, 2013.

16. Chen QH, Yang Y, He HL, Xie JF, Cai SX, Liu AR, Wang HL and Qiu HB: The effect of glutamine therapy on outcomes in critically ill patients: A meta-analysis of randomized controlled trials. Crit Care 18: R8, 2014.

17. Crowther M, Avenell A and Culligan DJ: Systematic review and meta-analyses of studies of glutamine supplementation in haematopoietic stem cell transplantation. Bone Marrow Transplant 44: 413-425, 2009.

18. Asrani V, Chang WK, Dong Z, Hardy G, Windsor JA and Petrov MS: Glutamine supplementation in acute pancreatitis: A meta-analysis of randomized controlled trials. Pancreatology 13: 468-474, 2013.

19. Zhong X, Liang CP and Gong S: Intravenous glutamine for severe acute pancreatitis: A meta-analysis. World J Crit Care Med 2: 4-8, 2013.

20. Tao KM, Li XQ, Yang LQ, Yu WF, Lu ZJ, Sun YM and Wu FX: Glutamine supplementation for critically ill adults. Cochrane Database Syst Rev: CD010050, 2014.

21. Wischmeyer PE, Dhaliwal R, McCall M, Ziegler TR and Heyland DK: Parenteral glutamine supplementation in critical illness: A systematic review. Crit Care 18: R76, 2014.

22. Oldani M, Sandini M, Nespoli L, Coppola S, Bernasconi DP and Gianotti L: Glutamine supplementation in intensive care patients: A meta-analysis of randomized clinical trials. Medicine (Baltimore) 94: e1319, 2015.

23. Yong L, Lu QP, Liu SH and Fan H: Efficacy of glutamine-enriched nutrition support for patients with severe acute pancreatitis: A meta-analysis. JPEN J Parenter Enteral Nutr 40: 83-94, 2016.

24. van Zanten AR, Dhaliwal R, Garrel D and Heyland DK: Enteral glutamine supplementation in critically ill patients: A systematic review and meta-analysis. Crit Care 19: 294, 2015.

25. Jiang $Z$, Jiang $H$ and Fürst $P$ : The impact of glutamine dipeptides on outcomes of surgical patients: Systematic review of randomized controlled trials from Europe and Asia. Clin Nutr Suppl 1: 17-23, 2004.

26. Zheng YM, Li F, Zhang MM and Wu XT: Glutamine dipeptide for parenteral nutrition in abdominal surgery: A meta-analysis of randomized controlled trials. World J Gastroenterol 12: 7537-7541, 2006.

27. Wang Y, Jiang ZM, Nolan MT, Jiang H, Han HR, Yu K, Li HL, Jie B and Liang XK: The impact of glutamine dipeptide-supplemented parenteral nutrition on outcomes of surgical patients: A meta-analysis of randomized clinical trials. JPEN J Parenter Enteral Nutr 34: 521-529, 2010.

28. Yue C, Tian W, Wang W, Huang Q, Zhao R, Li Q and Li J: The impact of perioperative glutamine-supplemented parenteral nutrition on outcomes of patients undergoing abdominal surgery: A meta-analysis of randomized clinical trials. Am Surg 79: 506-513, 2013.

29. Kang K, Shu XL, Zhang YS, Liu XL and Zhao J: Effect of glutamine enriched nutrition support on surgical patients with gastrointestinal tumor: A meta-analysis of randomized controlled trials. Chinese Med J (Engl) 128: 245-251, 2015.

30. Sandini M, Nespoli L, Oldani M, Bernasconi DP and Gianotti L: Effect of glutamine dipeptide supplementation on primary outcomes for elective major surgery: Systematic review and meta-analysis. Nutrients 7: 481-499, 2015.

31. Lin JJ, Chung XJ, Yang CY and Lau HL: A meta-analysis of trials using the intention to treat principle for glutamine supplementation in critically ill patients with burn. Burns 39: 565-570, 2013.

32. Mottaghi A, Yeganeh MZ, Golzarand M, Jambarsang S and Mirmiran P: Efficacy of glutamine-enriched enteral feeding formulae in critically ill patients: A systematic review and meta-analysis of randomized controlled trials. Asia Pac J Clin Nutr 25: 504-512, 2016.

33. Heyland DK, Dhaliwal R, Drover JW, Gramlich L and Dodek P; Canadian Critical Care Clinical Practice Guidelines Committee: Canadian clinical practice guidelines for nutrition support in mechanically ventilated, critically ill adult patients. JPEN J Parenter Enteral Nutr 27: 355-373, 2003. 
34. Newsholme P: Why is L-glutamine metabolism important to cells of the immune system in health, postinjury, surgery or infection? J Nutr 131 (9 Suppl): 2515S-2524S, 2001.

35. Shu XL, Yu TT, Kang K and Zhao J: Effects of glutamine on markers of intestinal inflammatory response and mucosal permeability in abdominal surgery patients: A meta-analysis. Exp Ther Med 12: 3499-3506, 2016.

36. Oliveira GP, Dias CM, Pelosi P and Rocco PR: Understanding the mechanisms of glutamine action in critically ill patients. An Acad Bras Cienc 82: 417-430, 2010.

37. Pradelli L, Povero M, Muscaritoli M and Eandi M: Updated cost-effectiveness analysis of supplemental glutamine for parenteral nutrition of intensive-care patients. Eur J Clin Nutr 69: 546-551, 2015

38. Rodas PC, Rooyackers O, Hebert C, Norberg A and Wernerman J: Glutamine and glutathione at ICU admission in relation to outcome. Clin Sci (Lond) 122: 591-597, 2012.

39. Baker GR, Norton PG, Flintoft V, Blais R, Brown A, Cox J, Etchells E, Ghali WA, Hébert P, Majumdar SR, et al: The Canadian adverse events study: The incidence of adverse events among hospital patients in Canada. CMAJ 170: 1678-1686, 2004.
40. Magill SS, Edwards JR, Bamberg W, Beldavs ZG, Dumyati G, Kainer MA, Lynfield R, Maloney M, McAllister-Hollod L, Nadle J, et al: Multistate point-prevalence survey of health care-associated infections. N Engl J Med 370: 1198-1208, 2014.

41. Klevens RM, Edwards JR, Richards CL Jr, Horan TC, Gaynes RP, Pollock DA and Cardo DM: Estimating health care-associated infections and deaths in U.S. hospitals, 2002. Public Health Rep 122: 160-166, 2007.

42. Heyland DK, Elke G, Cook D, Berger MM, Wischmeyer PE, Albert M, Muscedere J, Jones G and Day AG; Canadian Critical Care Trials Group: Glutamine and antioxidants in the critically ill patient: A post hoc analysis of a large-scale randomized trial. JPEN J Parenter Enteral Nutr 39: 401-409, 2015.

43. Vanek VW, Matarese LE, Robinson M, Sacks GS, Young LS and Kochevar M; Novel Nutrient Task Force, Parenteral Glutamine Workgroup; American Society for Parenteral and Enteral Nutrition (A.S.P.E.N.) Board of Directors: A.S.P.E.N. position paper: Parenteral nutrition glutamine supplementation. Nutr Clin Pract 26: 479-494, 2011. 